\title{
Optimal Any-Angle Pathfinding In Practice
}

\section{Daniel Harabor}

The University of Melbourne and

National ICT Australia, Victoria Laboratory

115 Batman St, Melbourne, 3003, Australia

\author{
Alban Grastien \\ National ICT Australia, Canberra Laboratory \\ 7 London Circuit, Canberra, 2601, Australia \\ Dindar $\ddot{O ̈}_{z}$ \\ Yasar University \\ Bornova, Izmir, 35100, Turkey
}

\author{
Vural Aksakalli \\ Istanbul Sehir University \\ Altunizade, Istanbul, 34662, Turkey
}

DANIEL.HARABOR@NICTA.COM.AU

ALBAN.GRASTIEN@NICTA.COM.AU

DINDAR.OZ@YASAR.EDU.TR

AKSAKALLI@SEHIR.EDU.TR

\begin{abstract}
Any-angle pathfinding is a fundamental problem in robotics and computer games. The goal is to find a shortest path between a pair of points on a grid map such that the path is not artificially constrained to the points of the grid. Prior research has focused on approximate online solutions. A number of exact methods exist but they all require super-linear space and pre-processing time. In this study, we describe Anya: a new and optimal any-angle pathfinding algorithm. Where other works find approximate any-angle paths by searching over individual points from the grid, Anya finds optimal paths by searching over sets of states represented as intervals. Each interval is identified on-thefly. From each interval Anya selects a single representative point that it uses to compute an admissible cost estimate for the entire set. Anya always returns an optimal path if one exists. Moreover it does so without any offline pre-processing or the introduction of additional memory overheads. In a range of empirical comparisons we show that Anya is competitive with several recent (sub-optimal) online and pre-processing based techniques and is up to an order of magnitude faster than the most common benchmark algorithm, a grid-based implementation of $A^{*}$.
\end{abstract}

\section{Introduction}

Any-angle pathfinding is a common navigation problem in robotics and computer games. It takes as input a pair of points from a uniform two-dimensional grid and asks for a shortest path between them that is not artificially constrained to the points of the grid. Such anyangle paths are desirable to compute as they are typically shorter than their grid-constrained counterparts and because following such a trajectory can give the appearance of realism and intelligence; e.g. to the player of a computer game. Despite its apparent simplicity any- 
angle pathfinding is surprisingly challenging. So far many successful and popular methods have been proposed, yet they all involve trade-offs of some kind. We begin with a few examples that highlight, in broad strokes, the main research trends and their limitations, to date.

In the communities of Artificial Intelligence and Game Development the any-angle pathfinding problem is often solved efficiently using a technique known as string pulling. The idea is to compute a grid-optimal path and then smooth the result; either as part of a post-processing step (e.g. Pinter, 2001; Botea, Müller, \& Schaeffer, 2004) or by interleaving string pulling with online search (e.g. Ferguson \& Stentz, 2005; Nash, Daniel, Koenig, \& Felner, 2007). Regardless of the particular approach, all string pulling techniques suffer from the same disadvantages: (i) they require more computation than just finding a path and; (ii) they only yield approximately shortest paths.

In the communities of Robotics and Computational Geometry a related and more general problem has been well-studied: finding Euclidean shortest paths between polygonal obstacles in the plane. Visibility Graphs (Lozano-Pérez \& Wesley, 1979) and the Continuous Dijkstra paradigm (Mitchell, Mount, \& Papadimitriou, 1987) are among the best known and most influential techniques that originate from this line of research. Even though both of these methods are optimal and efficient in practice they nevertheless suffer from having often undesirable properties: (i) the search graph ${ }^{1}$ must be pre-computed during an offline pre-processing step; (ii) if the map changes at any point the search graph is invalidated and must be recomputed, usually from scratch.

To date, it is not clear if there exists an any-angle pathfinding algorithm that is simultaneously online, optimal and also practically efficient (i.e. at least as fast in practice as grid-based pathfinding using $A^{*}$ search). In this manuscript, we present new work that answers this open question in the affirmative by introducing a new any-angle pathfinding algorithm called Anya. Our approach bears some similarity to existing works from the literature, most notably those algorithms based on the Continuous Dijkstra paradigm. In rough overview:

- Where other methods search over the individual nodes of the grid, Anya searches over contiguous sets of states that form intervals.

- Each Anya interval has a single representative point that is used to derive an admissible cost estimate (i.e $f$-value) for all points in the set.

- To progress the search process Anya projects each interval, from one row of the grid onto another, until the target is reached.

Anya always finds an optimal any-angle path, if one exists. In addition Anya does not rely on any pre-computation nor does it introduce any memory overheads (in the form of auxiliary data structures) beyond what is required to maintain an open and closed list. A theoretical description of this algorithm has previously appeared in the literature (Harabor \& Grastien, 2013). In this study we extend that work in several ways: (i) we give a

1. We distinguish between the search graph and the input grid map. Though in some contexts these terms coincide exactly this is not true in general. In particular the search graph may be a subset of the input grid or may be a related but entirely separate data structure. 



Figure 1: Examples of visible and non-visible pairs of points.

detailed conceptual description of the Anya algorithm and provide an extended theoretical argument for optimality and completeness; (ii) we discuss the practical considerations that arise when implementing the algorithm and we give a technical description for one possible and efficient implementation; (iii) we make detailed empirical comparisons showing that Anya is competitive with a range of recent sub-optimal techniques from the literature, including those based on offline pre-processing, and is up to one order of magnitude better than our benchmark grid-based implementation of $\mathrm{A}^{*}$; (iv) we discuss a range of possible extensions for further improving the current results.

\section{The Optimal Any-Angle Pathfinding Problem}

A grid is a planar subdivision consisting of $W \times H$ square cells. Each cell is an open set of interior points which are all traversable or all non-traversable. The vertices associated with each cell are called the discrete points of the grid. Edges in the grid can be interpreted as open intervals of intermediate points; each one representing a transition between two discrete points. Each type of point $p=(x, y)$ has a unique coordinate where $x \in[0, W]$ and $y=[0, H]$, with discrete points limited to the subset of integer $x$ and $y$ values.

A discrete or intermediate point is traversable if it is adjacent to at least one traversable cell. Otherwise it is non-traversable. A discrete point which is common to exactly four adjacent cells is called an intersection. Any intersection where three of the adjacent cells are traversable and one is not is called a corner. Two points are visible from one another if they can be connected by a straight-line path (i.e. a sequence of adjacent points, either intermediate or discrete) that does not: (i) pass through any non-traversable point or (ii) pass through an intersection formed by two diagonally-adjacent non-traversable cells. Figure 1 shows some examples that help to better illustrate this idea.

An any-angle path $\pi$ is a sequence of points $\left\langle p_{1}, \ldots, p_{k}\right\rangle$ where each $p_{i}$ is visible from $p_{i-1}$ and $p_{i+1}$. The length of $\pi$ is the cumulative distance between every successive pair of points $d\left(p_{1}, p_{2}\right)+\ldots+d\left(p_{k-1}, p_{k}\right)$. The function $d\left(p=(x, y), p^{\prime}=\left(x^{\prime}, y^{\prime}\right)\right)=\sqrt{\left(x-x^{\prime}\right)^{2}+\left(y-y^{\prime}\right)^{2}}$ is a uniform Euclidean distance metric. We will say $p_{i} \in \pi$ is a turning point if the segments $\left(p_{i-1}, p_{i}\right)$ and $\left(p_{i}, p_{i+1}\right)$ form an angle not equal to $180^{\circ} 2$. Finally, the any-angle pathfinding problem is one that requires as input a pair of discrete points, $s$ and $t$, and asks for an anyangle path connecting them. The point $s$ designates the source (equivalently, start) location

2. It is well-known that the turning points in optimal any-angle paths are corner points; e.g. as shown by Mitchell et al. (1987). 
while the point $t$ designates the target (equivalently, goal) location. Such a path is optimal if there exists no alternative any-angle path between $s$ and $t$ that is strictly shorter.

Figure 2 provides an example of an optimal any-angle pathfinding problem. As can be seen the source, target and all obstacles have discrete positions however the path itself does not need to follow the grid. Notice also that the trajectory of this path appears much more realistic than any alternative restricted to turning at modulo $45 \mathrm{deg}$ or $90 \mathrm{deg}$.

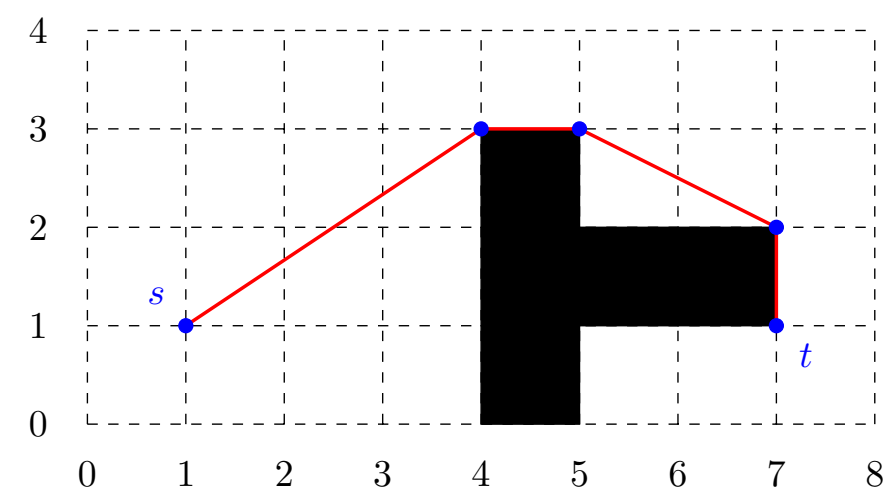

Figure 2: Example of an any-angle pathfinding problem together with its solution.

\section{An Overview of Anya}

Consider the any-angle instance shown in Figure 3. In this example the optimal path between $s$ and $t$ needs to first head towards the corner point $n$ and then change direction toward the target $t$. One possible approach to solving this problem involves computing a visibility graph: i.e., identifying all pairs of corners that are visible from one another, and also visible from the start and target locations, and then searching for a path on this graph. The main drawback in this case is that the visibility graph can be quite large (up to quadratic in the size of the grid) and very expensive to compute.

An alternative approach, which avoids these overheads, is to solve the problem online. Unfortunately online search methods generally consider only the discrete points of the grid and their immediate neighbours. For example, when expanding the point $s$ it is common to only generate the neighbours: $(1,0),(2,1)$, and $(3,0)$ in the example of Figure 3 . The A* $f$-value for each of the three neighbours is, respectively, $1+\sqrt{34} \simeq 6.83,1+\sqrt{20} \simeq 5.47$, and $1+\sqrt{26} \simeq 6.1$ (using Euclidean-distance as a heuristic). By comparison the optimal any-angle path has cost of $\sqrt{10}+\sqrt{5} \simeq 5$.4. Immediately we can see that the heuristic at hand does not satisfy one of the essential properties of $\mathrm{A}^{*}$ search: that the $f$-value of each node should always be an underestimate of the actual distance to the goal. Without this property $\mathrm{A}^{*}$ is not guaranteed to be optimal.

The issue described above comes from the fact that the optimal path does not go through any of the points $(1,0),(2,1)$, or $(3,0)$. Instead the optimal path crosses row 1 at point $y_{1}$, which is not part of the search space. To ensure optimality we should consider all points such as $y_{1}$ rather than just the discrete points of the grid. There are however many such points including e.g., points such as $y_{1}^{\prime}$ (leading to $(3,6)$ ), which apriori seems a reasonable candidate for expansion, but which does not appear on any optimal path. 


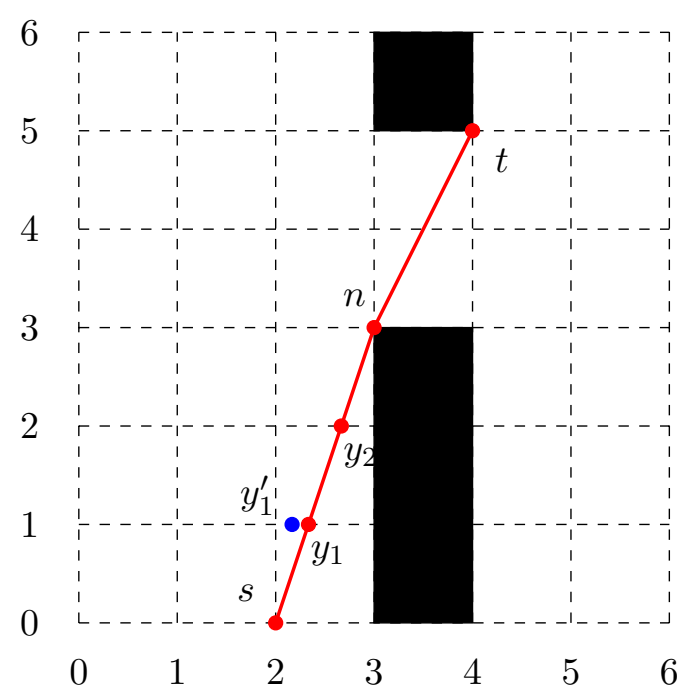

Figure 3: When pathfinding from $s$ to $n$, online algorithms such as $\mathrm{A}^{*}$ and Theta* only expand discrete points from the grid and never intermediate points such as $y_{i}$.

In general we need to consider all potential $y_{i}$ points defined as a fraction $\frac{h}{w}$ where $h \in\{0, \ldots, H\}$ and $w \in\{1, \ldots, W\}$. This set is quadratic in $n=\min (W, H)$. To understand why, we consider the Farey Sequence of order $n$, the sequence (ordered by increasing number) of all rational numbers between 0 and 1 that can be written as a fraction whose denominator is an integer lower than $n$. For instance, the Farey Sequence of order $n=6$ is: $0, \frac{1}{6}, \frac{1}{5}, \frac{1}{4}, \frac{1}{3}, \frac{2}{5}, \frac{1}{2}, \frac{3}{5}, \frac{2}{3}, \frac{3}{4}, \frac{4}{5}, \frac{5}{6}, 1$. Notice that $\frac{1}{3}=\frac{2}{6}$, which explains why the length of this sequence is not $n(n+1) \div 2$; still the asymptotic cardinality of this sequence is known to be $\frac{3 n^{2}}{\pi^{2}}$ (Graham, Knuth, \& Patashnik, 1989, ch. 9).

Since the quadratic behaviour of the Farey Sequence makes it impractical to enumerate all potential $y_{i}$ points we propose to consider, instead of individual points, a set of points that appear together as part of a contiguous interval on the grid. In the example of Figure 3 we would consider all the points lying between $(0,1)$ and $(3,1)$, at the same time and as part of a single $A^{*}$ search node. In this framework we need to:

- define formally an Anya search node,

- define the set of successors of a search node,

- define how to compute the $f$-value of a search node,

- prove optimality of the returned path,

- terminate search when no path is available,

- ensure the Anya algorithm is efficient in practice. 


\section{Algorithm Description}

This section presents in detail the Anya algorithm and its properties. Since Anya is a variant of $\mathrm{A}^{*}$ we first present its search space: the search nodes, the successors of a node and the evaluation function used to rank nodes during search. We then a give pseudo-code description of the algorithm and discuss its properties. Improvements that make Anya efficient in practice are presented in the next section.

\subsection{Anya Search Nodes}

We now define the notion of interval, which is at the core of Anya.

Definition $1 A$ grid interval $I$ is a set of contiguous and pairwise visible points drawn from any discrete row of the grid. Each interval is defined in terms of its endpoints a and $b$. With the possible exception of $a$ and $b$, each interval contains only intermediate and discrete non-corner points.

By definition, all points in an interval share the same $y$ position, which is a positive integer. Moreover, the $x$ position of all points in an interval (including that of endpoints $a$ and $b$ ) is a rational number ${ }^{3}$. We will use normal parentheses "(" or ")" to indicate an interval endpoint that is open and square brackets "[" or "]" to indicate an interval endpoint that is closed. For example, the interval $I=(a, b]$ is open at (i.e. does not include) $a$ and closed at (i.e. does include) $b$.

Identifying intervals is simple: any row of the grid can be naturally divided into maximally contiguous sets of traversable and non-traversable points. Each traversable set forms a tentative interval which we can split, repeatedly if necessary, until all corner points are end points of intervals. Intervals can also be identified through an operation called projection. We discuss this procedure in the next sub-section. For now we note only that intervals produced by way of projection can also have non-discrete and non-corner endpoints.

A significant advantage of Anya is that we construct intervals on-the-fly. This allows us to start answering queries immediately and for any discrete start-target pair. Similar algorithms, e.g. Continuous Dijkstra (Mitchell et al., 1987), require a pre-processing step before any queries can be answered and then only from a single fixed start point.

Definition $2 A$ search node $(I, r)$ is a tuple where $r \notin I$ is a point called the root and $I$ is an interval such that each point $p \in I$ is visible from $r$. To represent the start node itself, set $I=[s]$ and assume $r$ is located off the plane and visible only from $s$; the cost from $r$ to $s$ in this case is zero.

An $A^{*}$ search node, together with its parents, traditionally represents the single path defined by travelling in straight line between the points in the search nodes from the root to the current node. An Anya search node similarly defines paths obtained by visiting the roots of each nodes and ending in the interval of the current node. A node therefore represents many paths and the root of the search node is always the last (common) turning

3. As per the problem definition, every point $(x, y)$ appearing on an optimal any-angle path belongs to a Farey Sequence and all such points are rational. 
point of these paths: it will always be either the root of the parent node or one of the end points of the parent interval.

Besides the start node, which we treat as a special case, there are two other types of search nodes: cone nodes and flat nodes. An example of a cone node is shown in Figure 4. Such nodes are characterised by the fact that the root $r$ is not on the same row as its associated interval $I$. Notice in the example that although the interval $I=[a, b]$ is maximal, it does not have any endpoints which are obstacles, corners or indeed even discrete points of the grid (here the left endpoint $a$ is $(2.5,4)$ while the right endpoint $b$ is $(5.5,4))$. Examples of flat nodes are shown in Figure 5. The two nodes are: $\left(\left(a_{1}, b_{1}\right], r\right)$ and $\left(\left(a_{2}, b_{2}\right], r\right)$. Flat nodes are characterised by the fact that the root $r$ is on the same row as the interval $I$. Notice in the examples given that $a_{1}=r$ (resp. $a_{2}=b_{1}$ ) is excluded from the first (resp. second) interval. The semantics of every search node is that the current position is located somewhere in the interval $I$ and we reach that point by an any-angle path whose most recent turning point is $r$.

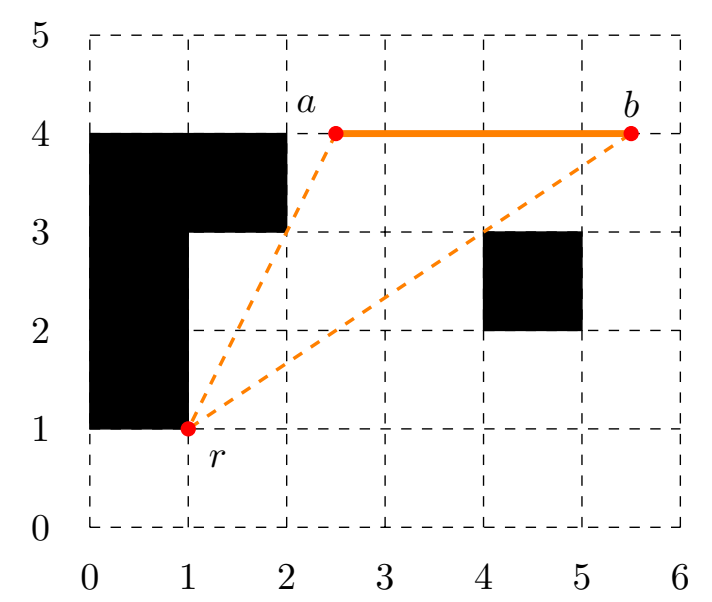

Figure 4: Example of a cone search node.

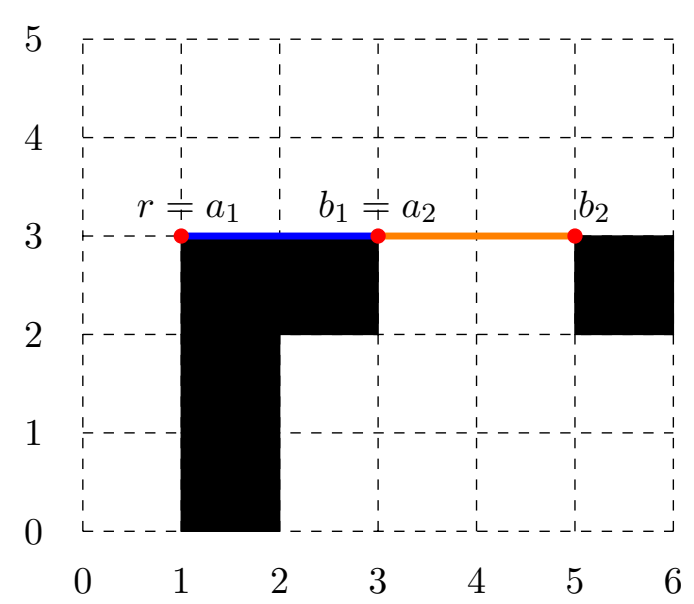

Figure 5: Example of two flat search nodes.

\subsection{Searching with Anya: Successors}

The successors of a search node $n$ are identified by computing intervals over sets of traversable points; from the same row of the grid as the current node $n$ and from the rows immediately adjacent. We want to guarantee that each point in such a set can be reached from the root of $n$ via a local path which is taut. Taut simply means that if we "pull" on the endpoints of the path we cannot make it any shorter. We now provide a formal definition of a successor and then discuss how this definition can be applied in practice.

Definition $3 A$ successor of a search node $(I, r)$ is a search node $\left(I^{\prime}, r^{\prime}\right)$ such that

1. for all points $p^{\prime} \in I^{\prime}$, there exists a point $p \in I$ such that the local path $\left\langle r, p, p^{\prime}\right\rangle$ is taut;

2. $r^{\prime}$ is the last common point shared by all paths $\left\langle r, p, p^{\prime}\right\rangle$; and

3. $I^{\prime}$ is maximal according to the points above and the definition of a search node. 
The first requirement (tautness) implies that each successor $p^{\prime} \in I^{\prime}$ can be reached from the root of the current node $r$ by a path that is locally optimal. We will use this property in the next subsection to show that Anya always finds a globally optimal path if one exists at all. The third property, requiring that each successor have an interval that is maximal, exists for the purpose of practical efficiency: simply put, we do not want to have arbitrarily small and arbitrarily many successors. Instead, we will make each successor interval as large as possible. The second property has two interpretations. When $r^{\prime}=r$ we will say that the successor node is observable. Similarly when $r^{\prime}=p$ we will say that the successor is non-observable. We explore each of these ideas in turn.

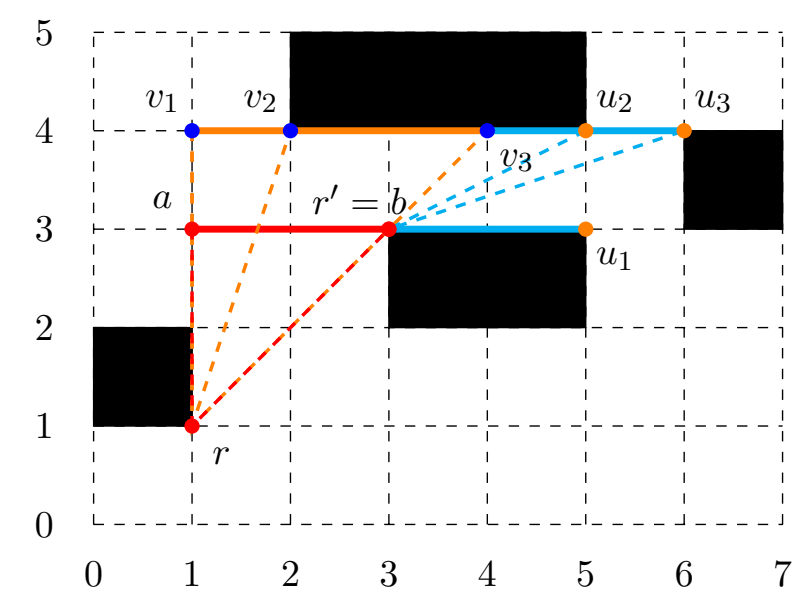

Figure 6: Successors of a cone search node, $n=([a, b], r)$. There are five successors: $\left(\left[v_{1}, v_{2}\right], r\right)$ and $\left(\left(v_{2}, v_{3}\right], r\right)$ which are observable and $\left(\left(r^{\prime}, u_{1}\right], r^{\prime}\right),\left(\left(v_{3}, u_{2}\right), r^{\prime}\right)$, and $\left(\left[u_{2}, u_{3}\right], r^{\prime}\right)$ which are not.

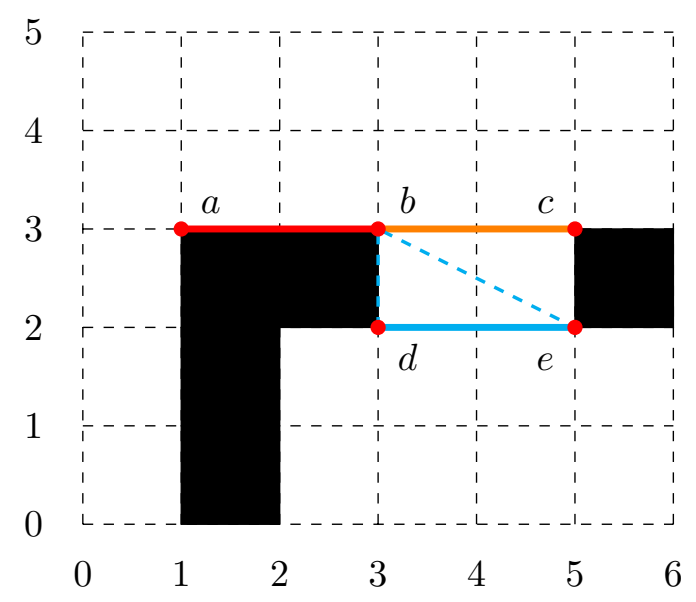

Figure 7: Successors of a flat search node, $n=((a, b], a)$. There are two successors: $((b, c], a)$ which is observable and $([d, e], b)$ which is not. 


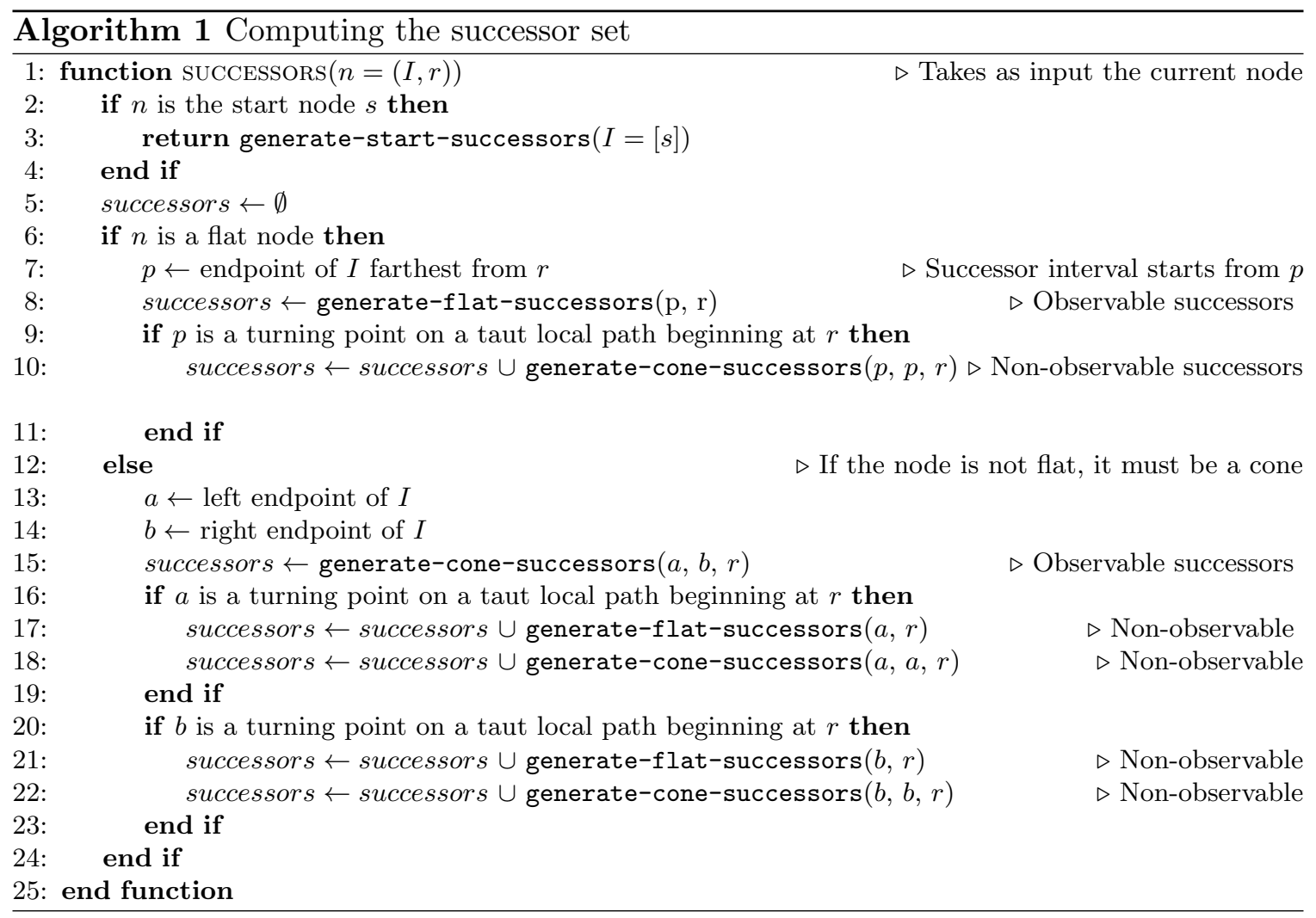

An observable successor is characterised by the fact that all points $p^{\prime} \in I^{\prime}$ are visible from the current root point $r$. In this case the last common point shared by all local paths of the form $\left\langle r, p, p^{\prime}\right\rangle$ is $r$. Observable successors are computed by projecting the current interval on the next row. The projection identifies a maximal interval $I_{\max }$ that we will split at each internal corner point point. Each interval produced by the split operation leads to a new observable successor, and all such successors share the same root point as the original (parent) node. This process is illustrated in Figure 6 where the interval $I=[a, b]$ is projected onto the next row. The projection identifies a maximal observable interval $I_{\max }=\left[v_{1}, v_{3}\right]$ which is subsequently split to create two observable successors: $\left(\left[v_{1}, v_{2}\right], r\right)$ and $\left(\left(v_{2}, v_{3}\right], r\right)$.

By comparison, a non-observable successor is characterised by the fact that all points $p^{\prime} \in I^{\prime}$ are not visible from the current root $r$. In this case all local paths of the form $\left\langle r, p, p^{\prime}\right\rangle$ must pass through a (visibility obstructing) corner point whose identity is $r^{\prime}:=p$. Figure 6 illustrates the process of computing non-observable successors. First, from the non-observable points to the right of the current interval $I=[a, b]$, we construct a single flat successor with $I^{\prime}=\left(b, u_{1}\right]$ and root $r^{\prime}:=b$. Non-observable points also exist to the left of the current interval $I$ but the local path to each such point (from $r$ through $a$ ) is not taut. Other non-observable successors can be found on rows of the grid adjacent to the current interval $I$. By projecting the corner endpoint $b$ onto the next row of the grid we can construct two further non-observable successors: $\left(\left(v_{3}, u_{2}\right), b\right)$ and $\left(\left[u_{2}, u_{3}\right], b\right)$.

In Algorithm 1 we give an overview of the procedure that generates the successor set for each search node. An overview of the sub-functions appearing in Algorithm 1 is given in 
the appendix. The implementation is straightforward, requiring nothing more complicated than grid scanning operations and linear projections.

It is important to note at this stage that Anya does not perform any visibility checks during the generation of successor nodes. Visibility checks are at the heart of many contemporary online algorithms, including Theta* (Nash \& Koenig, 2013), which must determine whether each successor is visible from some other node (e.g. the grand-parent node). On the one hand visibility checks help Theta* et al. find shorter paths and expand fewer nodes than traditional $\mathrm{A}^{*}$ search. On the other hand, the computational overhead introduced by these checks means that run-times can often be larger than $A^{*}$. By comparison when Anya projects an interval $I$, from one row of the grid to the next, the process involves only local reasoning. In particular we can determine if the projection $I_{\max }$ is valid, invalid or if it needs to be clipped by simply testing the traversability of cells located above, below and to the left and right of the current interval $I$ and the proposed $I_{\max }$. The elimination of visibility checks is an important practical advantage for Anya. As we will see in Section 9, Anya not only finds shorter paths than online methods such as Theta* et al. it is also usually much more efficient in terms of running time.

We now illustrate Algorithm 1 using previous examples. Consider the flat node $((a, b], a)$ of Figure 7 . The point $p$ of Line 7 is set to $b$ and the observable flat successor $((b, c], a)$ is generated on Line 8. Furthermore since $b$ is a turning point from $a$ (Line 9), the interval $I_{\max }=[d, e]$ is considered. Since $I_{\max }$ contains no interior corner points it is not split and a single non-observable cone successor $\left(I=I_{\max }, b\right)$ is generated (Line 10).

Next, consider the cone node $([a, b], r)$ of Figure 6 . First we generate the observable successors (Line 15): the interval $[a, b]$ is projected and the maximal interval $I_{\max }=\left[v_{1}, v_{3}\right]$ is identified. $I_{\max }$ is then split at the internal corner point $v_{2}$ leading to two observable cone successor nodes, $\left(I_{1}=\left(v_{1}, v_{2}\right], r\right)$ and $\left(I_{2}=\left(v_{2}, v_{3}\right], r\right)$. Notice that no line-of-sight visibility check is required here. Next since $b$ is a turning point we look for non-observable successors as well (Lines 20-22). The flat successor $\left(\left(b, u_{1}\right], b\right)$ is generated as per the previous example. Meanwhile the maximal (non-observable) cone interval $I_{\max }=\left(v_{3}, u_{3}\right]$ is also identified. This interval is split at the internal corner point $u_{2}$ resulting in two non-observable cone successor nodes, $\left(I_{3}=\left(v_{3}, u_{2}\right], b\right)$ and $\left(I_{4}=\left(u_{2}, u_{3}\right], b\right)$.

Algorithm 1 treats the start node (Lines 2-4) as a special case because its root point is located off the grid. The successors of the start node (i) are all non-observable intervals from the root and (ii) can be found to the left and right of the start location, on the row immediately above the start location and on the row immediately below.

\subsection{Evaluating an Anya Search Node}

The search procedure of Anya, similarly to that of A*, always expands the "most promising" node found so far. It is therefore necessary to evaluate each root and interval pair. This evaluation corresponds to an estimate $f$ of the minimal length of a path from the source to the target through the current interval. An optimality condition of $\mathrm{A}^{*}$ is that this estimate is optimistic (i.e. it is never larger than the actual optimal path length). In classical $\mathrm{A}^{*}$ where a search node $n$ corresponds to a single point $p$ on the grid the value $f(n)$ is computed as the sum of $g(p)$, the length of the path from the source to $p$, and $h(p)$, an (under)estimation of the length of the shortest path from $p$ to the target. 
As a search node $n=(I, r)$ represents a set of points its $f$ value is the minimum $f$ value of the points in the node:

$$
f(n)=\inf _{p \in I} f(s, r, p, t)
$$

where $f(s, r, p, t)$ is an (under)estimate of the shortest path from $s$ to $t$ through $r$ and $p$. It should be noted that, because the set of points $p$ is continuous and potentially open, the minimum is replaced by the infimum. Since all points in the interval are visible from $r$, this value can be broken down as follows:

$$
f(s, r, p, t)=g(r)+d(r, p)+h(p)
$$

where $d(r, p)$ is the distance between the points $r$ and $p$.

Finding the point of the interval that minimises the $f$ value may seem like a hard problem since the interval contains a large number of points and we want to avoid generating all of them. However the straight-line distance heuristic $h(h(p)=d(p, t))$ makes it easy to isolate the point $p$ that minimises the $f$ value, thanks to two simple geometric observations. More precise heuristics are available but these could make it harder to find the point $p$.

Lemma 1 Let $t$ and $r$ be two points s.t. the interval $I$ is on the same row as $t$ or on a row between the rows of $r$ and $t$. Then the point $p$ of I with infimal $f$-value is the point in $I$ closest to the intersection of the straight-line path $\langle t, r\rangle$ with the row of $I$.

If the line between $r$ and $t$ intersects the interval then the point $p$ is the intersection. Otherwise this point $p$ is one of the endpoints of the interval. In the event that the precondition of Lemma 1 is not satisfied, it is possible to replace $t$ by its mirrored version $t^{\prime}$ through $I$ and thus satisfy the precondition. This case is described in Lemma 2.

Lemma 2 The mirrored point $t^{\prime}$ of target $t$ through interval $I$ is such that $d(p, t)=d\left(p, t^{\prime}\right)$ for all $p \in I$.

Lemma 2 is a trivial geometrical result. Both lemmas are illustrated on Figure 8.

\subsection{Search Procedure}

The search procedure employed by Anya is presented in Algorithm 2. It follows the pattern of $\mathrm{A}^{*}$ and uses priority queue, open, that stores all the yet-to-be-expanded search nodes ordered by $f$ value. Each node stores a pointer to its parent. At each step of the search Anya extracts the best node from open and checks if the corresponding interval contains the target. In the event that the target is found (Line 6) the returned path is a sequence of root points constructed by following back-pointers, from the current node to the start location. If the target is not found the current node is expanded and its successors are added to the priority queue (Line 8). Some successors may be considered redundant and these can be safely discarded without insertion into the priority queue (Line 9). We discuss this aspect of the algorithm in Section 6; for now it suffices to know that such successors are not on any optimal path. The expansion process continues until the target is found or the open list is exhausted, in which case the algorithm returns failure (Line 14).

In the next sections we will prove some fundamental properties about this algorithm: correctness, optimality and completeness. 


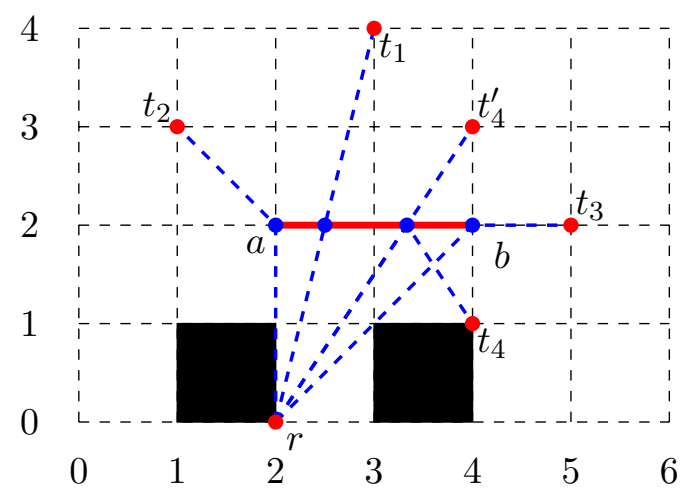

Figure 8: An illustration of Lemmas 1 and 2. We evaluate the node $n=([a, b], r)$. The points $t_{1}$ and $t_{4}^{\prime}$ correspond to the case where the row of the target $t$ intersects the interval $I ; t_{2}$ and $t_{3}$ where it does not; $t_{4}$ where the mirrored target $t_{4}^{\prime}$ must be used.

\section{Correctness and Optimality}

In this section we prove that Anya is correct and always finds an optimal path. In particular we will show (i) that the optimal path appears in the search space, (ii) when the target is expanded we have found an optimal path, and (iii) that each node in the search space will be reached in a finite number of steps. The topics of termination and completeness are discussed in Section 6.

We begin the analysis by recalling that a search node $n=(I, r)$ represents a set of potential paths (from $s$ to $r$ and from $r$ to each point $p \in I$ ). Following these semantics we will say that $n$ is a search node of a path $\pi$ if $r \in \pi$ and $I$ intersects $\pi$.

Lemma 3 If $n=(I, r)$ is a search node of an optimal path $\pi^{*}$ then: either $n$ contains the target $t$ or $n$ has at least one successor $n^{\prime}$ that is also a search node of $\pi^{*}$.

Proof: Start node: $n$ is the start node with $I=[s]$ and $r$ located off the grid. Additionally, $n$ is a search node of $\pi^{*}$ (hypothesis). Algorithm 1 (Line 3) scans the traversable




points of the grid that are visible from and adjacent to $s$. These points are located to the left and right of $s$ and they are located on the rows immediately above and immediately below the row of $s$. Algorithm 1 assigns each of these points to an interval $I^{\prime}$ and each $I^{\prime}$ is associated with a successor node that has as its root $r^{\prime}=s$. Every optimal path must pass through $I=[s]$ and there are no traversable points that can be reached from $s$ without passing through an interval associated with a successor of $s$. This is sufficient to satisfy the lemma.

Other nodes: $n$ is an arbitrary node of $\pi^{*}$ and $t \notin I$ (if $t \in I$ there are no further successors and we are done). By definition $r \in \pi^{*}$ and $p \in I$ is the (apriori unknown) intersection of $\pi^{*}$ with the interval $I$. There are now two possibilities to consider, depending on whether $p$ is a turning point or not. We will show that in both cases there is a successor of $n$ whose interval $I^{\prime}$ intersects $\pi^{*}$, which is sufficient to satisfy the lemma.

Case $1 p \in I$ is not a turning point. Algorithm 1 (Lines 8 and 15) scans all points that are adjacent to $I$ and (straight-line) visible from $r$ through $I$. Each point is assigned to a successor with observable interval $I^{\prime}$ and root point $r$. Thus at least one of the successors of $n$ intersects every straight line path from $r$ through $p$ which means at least one successor of $n$ intersects $\pi^{*}$.

Case $2 p \in I$ is a turning point. In this case $p$ must be a corner endpoint of $I$, otherwise $\pi^{*}$ is not taut and thus cannot be optimal. Algorithm 1 (Lines 10, 17, 18, 21) scans all points that are adjacent to $I$ and reachable from $r$ through $p$ by a taut local path. These points are located on the row of $p$ or on a row that is immediately adjacent. Each such point is assigned to a successor with a non-observable interval $I^{\prime}$ and root $r=p$. As the process is exhaustive all points reachable by a taut local path, from $r$ though $p$, must be assigned to an interval. Thus $\pi^{*}$ must intersect at least one of the successors of $n$.

Corollary 4 If there is a path between the source and the target, then the open list always contains a search node of an optimal path (or this node is currently being processed).

Proof: By induction.

base case: The initial search node is a node of any path from $s$.

inductive case: assume that the open list contains a search node of an optimal path. Then this node can only be removed if it is expanded. If this node does not contain the target then we know from Lemma that one successor will be generated that is a search node of this optimal path. Therefore a new search node of an optimal path will be inserted in the open list.

Lemma 5 The first expanded node that contains the target $t$ corresponds to one optimal path to $t$. 
Proof: Sketch. First we notice that the $f$-value of a node is indeed the minimal value of all the nodes in the interval, which means that $f$ is an under estimate $(\leq)$ of the actual cost to the target. Second we notice that, given a search node $(I, r)$ and its successor $\left(I^{\prime}, r^{\prime}\right)$, for each point $p^{\prime} \in I^{\prime}$, the $f$-value of $p^{\prime}$ is greater than or equal to the $f$-value of some point $p \in I\left(p=r^{\prime}\right.$ if $r^{\prime} \neq r ; p$ is the intersection of $I$ and $\left(r, p^{\prime}\right)$ otherwise); the $f$ function is therefore monotonically increasing. Finally, the $f$ function of a search node $(I, r)$ is the length of the path if $t \in I$. Hence the $f$ function of the nodes representing a sub-optimal path to $t$ will eventually exceed the optimal path distance, while the $f$ function of the nodes representing the optimal path will always remain under this value.

Lemma 6 If the target is reachable Anya will eventually expand a node whose interval includes the target.

Proof: By contradiction, assume that Anya does not expand a node whose interval includes the target. From Lemma 5 we know that failure to expand this node means that Anya expand infinitely many nodes. We shall prove that doing so implies that the $f$ value of these nodes is unbounded and, therefore, the target is not reachable.

For most search nodes $\left(I^{\prime}, r^{\prime}\right)$, the interval $I^{\prime}$ is on a different row than that of its parent $(I, r)$. Therefore, for those nodes, the value $g\left(p^{\prime}\right)$ is larger than the value $g(p)$ by 1 or more. This does not happen when the node is flat, but there can be only a bounded number of successive flat nodes. ${ }^{4}$ Hence an infinite sequence of successive Anya nodes has an infinite length. Finally each Anya node has a bounded number of successors, meaning that an infinite number of expansions will have to generate an infinite number of successive nodes.

\section{Completeness and Termination}

Until now we have not specified any policy by which Anya can detect nodes that have been previously expanded. In the context of optimal $A^{*}$ search such a policy is essential to prevent cyclical re-expansion and to ensure that the algorithm eventually terminates, even if there is no path between the start and target locations. In this section we describe such a policy for Anya. Conceptually similar to the A* closed list our approach works by tracking the best $g$-value associated with every root location (cf. every search node) encountered during search.

As a motivating example consider Figure 9 where the root $r$ is reached via two paths of different length. In the example the green path is strictly longer than the red path and any points reached via the green path will have a $g$-value that is strictly larger than the same point when reached via the red path. Figure 10 shows a similar example where both green and red paths reach the root point $r$ with the same cost, resulting in two identical copies of the successor node $(I, r)$. Without any strategy to handle such root-level redundancies the search process can generate many unnecessary nodes that slow progress to the goal. Moreover, if there exists no path between the start and target location, the search may not

4. And, furthermore, the value $g(p)$ does not increase significantly only for an unobservable flat cone. 


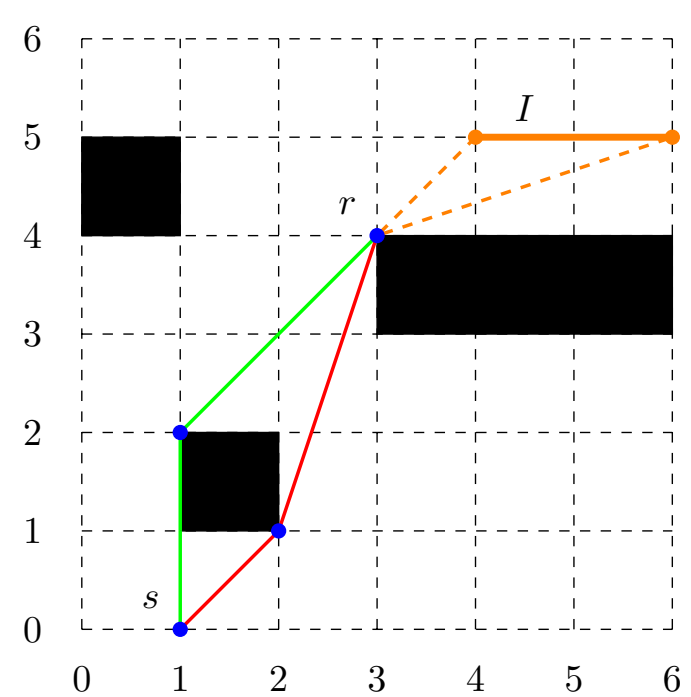

Figure 9: Root $r$ is reached via two paths of different lengths.



Figure 10: Root $r$ is reached via two paths of equal length.

terminate (e.g. when the input graph contains cycles it is possible to endlessly generate copies of states with ever increasing $g$-values).

We propose the following strategy to avoid root-level redundancies:

1. We store a hash table of all visited roots with their best $g$-values. We call this table the root history and apply it in a similar way to (and indeed in lieu of) a traditional $\mathrm{A}^{*}$ closed list.

2. When generating a search node $n$ we check if its root is already in the root history with a $g$-value less than or equal to its current $g$-value.

3. If the current $g$-value of the root improves on the value stored in the root history we add the node to the open list. We also update the $g$-cost of the $\operatorname{root}^{5}$ in the root history list.

4. Alternatively, if the current $g$-value of the root does not improve on the value stored in the root history we simply discard the node (i.e. it is not added to open).

The root history is implemented as a hash table. Its size is $O(n)$ where $n$ is the number of discrete points on a given input map. We now show that keeping a root history list does not affect the correctness or optimality of search and that Anya is indeed complete and does terminate.

Lemma 7 Anya search prunes only sub-optimal paths.

5. Similar updates to nodes on the closed list are sometimes performed in the context of incremental, bounded cost or bounded sub-optimal search. Such updates are performed as part of an operation called node re-opening. Our updates are not the same as node re-opening. In particular root points are never directly expanded and thus never appear on the open list (Anya search comprise root-interval pairs). 
Proof: Trivial. If a search node has a root with a sub-optimal $g$ value, then it represents a sub-optimal path.

\section{Lemma 8 Anya always terminates.}

Proof: For Anya to not terminate, it must explore paths of arbitrary length. Such paths must eventually involve the same root twice with the root being different in-between. Let $n$ and $n^{\prime}$ be the two such search nodes. The $g$ value associated with $n^{\prime}$ must be higher than the $g$ value associated with $n$ and, therefore, node $n^{\prime}$ must be pruned. Indeed all sufficiently long paths will be pruned and the open list will eventually be empty.

Lemma 9 Anya with redundant node pruning keeps at least one optimal path.

Proof: If a search node $n=(I, r)$ is removed there exists another search node $n^{\prime}$ (but with different search parents) with a smaller (or equal) $g$-value that is kept. Assume that $n$ is a search node of an optimal path $p_{1}, \ldots, p_{k}$, and let $p_{i} \in I$ be the point of this path that intersects $I$. Since the $g$-value of $n$ is similar to that of $n^{\prime}$, there exists another path $p_{1}^{\prime}, \ldots, p_{i}^{\prime}, p_{i+1}, \ldots, p_{k}$ of similar length, and this path is not pruned.

\section{Practical Pruning Strategies}

A* orders nodes for expansion by evaluating and ranking how promising they each appear (i.e. by their $f$-values). It is, however, possible to alter the order of expansion without compromising the guarantees provided by $\mathrm{A}^{*}$ : correctness, optimality and completeness. Indeed such a strategy can even have a positive effect on the efficiency of the overall search. In this section we discuss two practical strategies that modify expansion order and speed up search. Both enhancements are applied on-the-fly and both focus on reducing the size of the priority queue. The first strategy, Cul-de-sac Pruning, identifies nodes that can be safely discarded because they cannot possibly lead to the goal. The second strategy, Intermediate Pruning, is similar but works by avoiding the explicit generation of nodes that have only a single successor (these successors are expanded immediately, without being added to the open list).

\subsection{Cul-de-sac Pruning}

One way of reducing the size of the priority queue involves the early identification of $c u l$ de-sacs (cds). A cds is a search node that has no successor and does not contain the target. By definition a cds does not need to be added to the open list since its expansion cannot lead to the target. A simple test to identify cds nodes is given in Algorithm 3 by way of the procedure IS-CUL-DE-SAC.

Early pruning of cds nodes speeds up search (and reduces required memory) by preventing some unnecessary operations on open and also by reducing the size of the list, which 


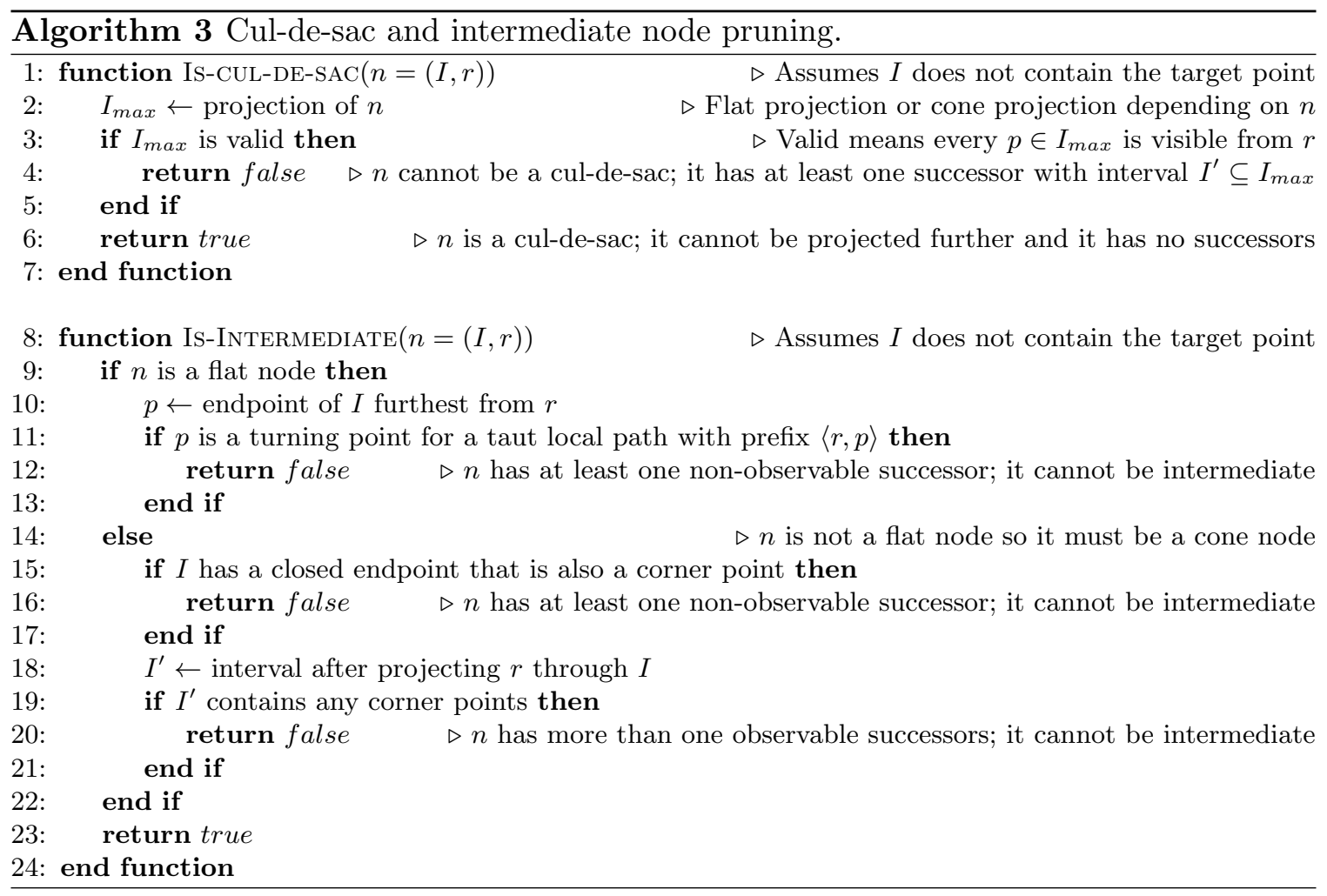

makes every other operation faster. For reference, when the open list is implemented as a binary heap each add or remove operation has a time complexity of $\log n$, where $n$ is the size of the list. Examples of cds pruning, for cone nodes and for flat nodes, are illustrated in Figure 11 and Figure 12. In both cases the current node and root are shown in blue while the intervals in red can be pruned.

\subsection{Intermediate Pruning}

Our second pruning strategy can be described as "pushing" the expansion in one direction as far as possible as long as it does not increase the branching factor. Practically, if a search node is generated that is guaranteed to have only one successor, then we immediately generate this successor instead of the originally intended node. If said successor also has only one successor the process can be recursively applied. Examples showing the application of intermediate pruning are given in Figure 13 for cone nodes and on Figure 14 for flat nodes. A simple test to identify intermediate nodes is given in Algorithm 3 by way of the procedure IS-INTERMEDIATE.

The first obvious benefit of intermediate pruning is a reduction in the number of operations on the open list. However a second benefit is that pushing the expansion of a node can lead to a cul-de-sac. When this happens no node at all is added to the open list, which helps keep the size of this list small and the operations on this list fast.

A potential issue with Intermediate Pruning is that that recursive application to nonpromising successor nodes could be more costly (in terms of time) than simply adding those 


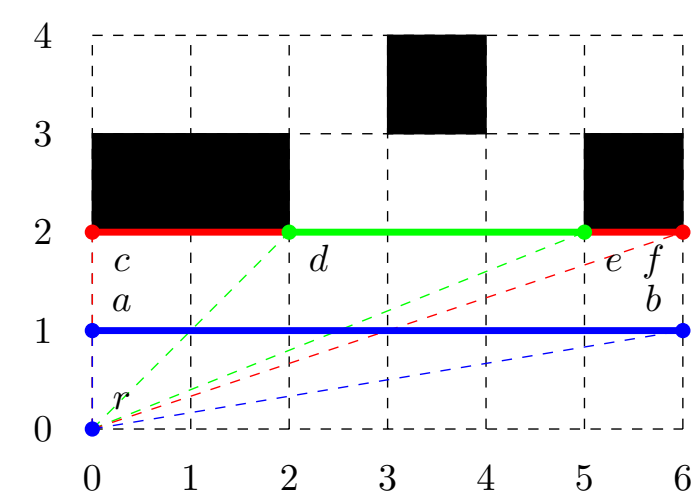

Figure 11: Cul-de-sacs in cone nodes: nodes $([c, d), r)$ and $((e, f], r)$ are not generated.

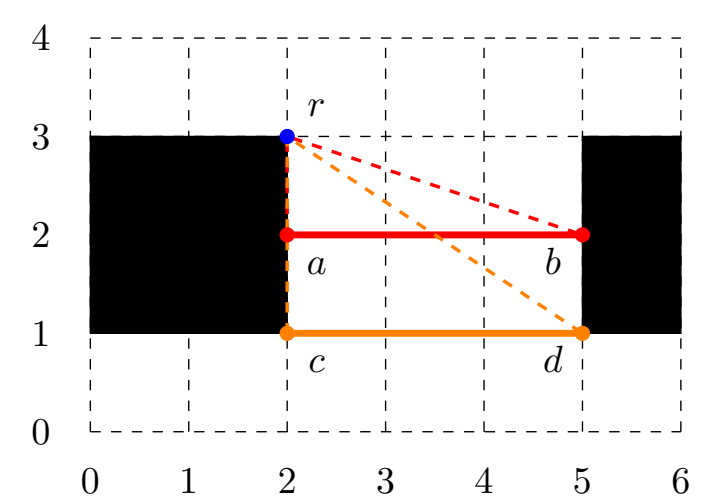

Figure 13: Intermediate node $([a, b], r)$ has only one successor, $([c, d], r)$, which is immediately generated.

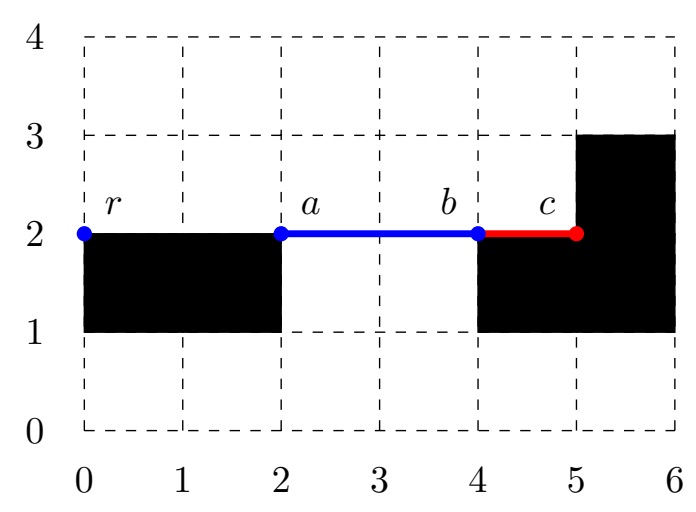

Figure 12: Cul-de-sac on flat nodes: node $((b, c], r)$ is not generated.

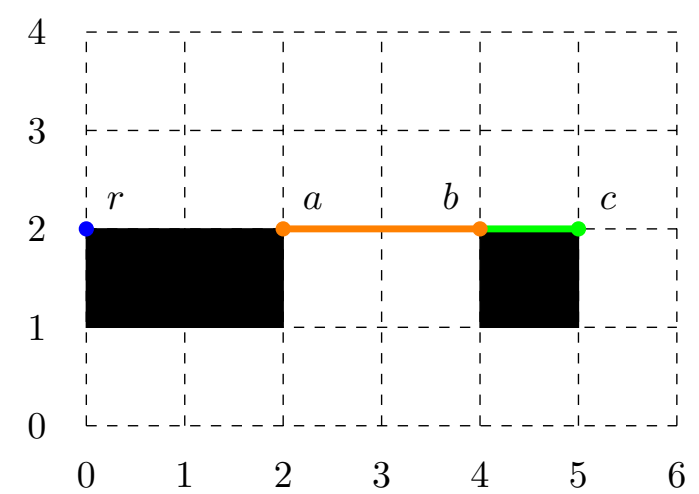

Figure 14: Intermediate node $([a, b], r)$ has only one successor, $((b, c], r)$ which is immediately generated.

nodes to open. We discuss this issue in more detail in Section 7.3. We also note that in our run-time experiments the application of Intermediate Pruning has a net positive effect on the performance of search.

\subsection{Discussion}

We have introduced two different ways in which nodes from the frontier of search can be pruned: Cul-de-sac Pruning and Intermediate Pruning. Both modify the expansion order of search and both improve performance along a single fixed path. They do this by pruning away sterile branches and by skipping over intermediate locations where no actual branching occurs. Similar strategies have been previously discussed in the literature. For example Culde-sac Pruning is based on the same set of principles as the Dead-end Heuristic (Björnsson \& Halldórsson, 2006); although our method reasons more locally and is applied purely online. Intermediate Pruning shares some similarities with Fast Expansion (Sun, Yeoh, Chen, \& 
Koenig, 2009); the main difference is that we prune nodes without reference to their $f$-value. Intermediate Pruning is also similar to Jump Point Search (Harabor \& Grastien, 2014) but applied outside the context of symmetry breaking and extended to sets of points taken as intervals rather than applied over the individual cells of the grid.

Anya's root history list, discussed in Section 6, can also be regarded as a type of pruning enhancement. In this case we reason more generally about the set of all possible paths that could be used to reach a given point and prune away only those successors that cannot possibly be on any optimal path. The approach we have taken here is similar in principle (but not in practice) to the pruning of redundant states in real-time search (Sturtevant \& Bulitko, 2011).

Pruning search nodes in Anya is more difficult than in classical A* search and its many modern progenitors. This is because each Anya node represents a set of positions rather than just one. Consider the example of Figure 15; we are particularly interested in the interval $[a, b]$ which can be generated with root $r_{1}$ or $r_{2}$. The shortest path from $s$ to $t$ is through $r_{1}\left(\sim 14.24\right.$ against $\sim 14.99$ for $\left.r_{2}\right)$. However if an obstacle is put on the cell labeled with $O$, then the optimal path switches to $r_{2}(\sim 15.62$ against $\sim 15.94)$. The diagram suggests that, when given the target and two search nodes sharing the same interval, it may not be possible to prune either of them.

The situation described in Figure 15 is not uncommon in practice and such examples may motivate us to derive new and more sophisticated pruning rules to further enhance the performance of the Anya algorithm. We must be careful however to weigh the improved pruning power of such new techniques against the overhead of applying them in the first instance. For example, an alternative (arguably, better) approach to avoiding redundant node expansions is to keep an interval history list in addition to (or instead of) a root history. Such a method would certainly avoid the problem outlined in Figure 15 but there are many more possible intervals than roots, which means the size of the hash table is potentially much larger and memory accesses are potentially slower. Additionally, comparing intervals for equality and membership requires extra time and may not be worth the investment ${ }^{6}$.

6. We attempted a similar experiment but the results were not clearly positive. 


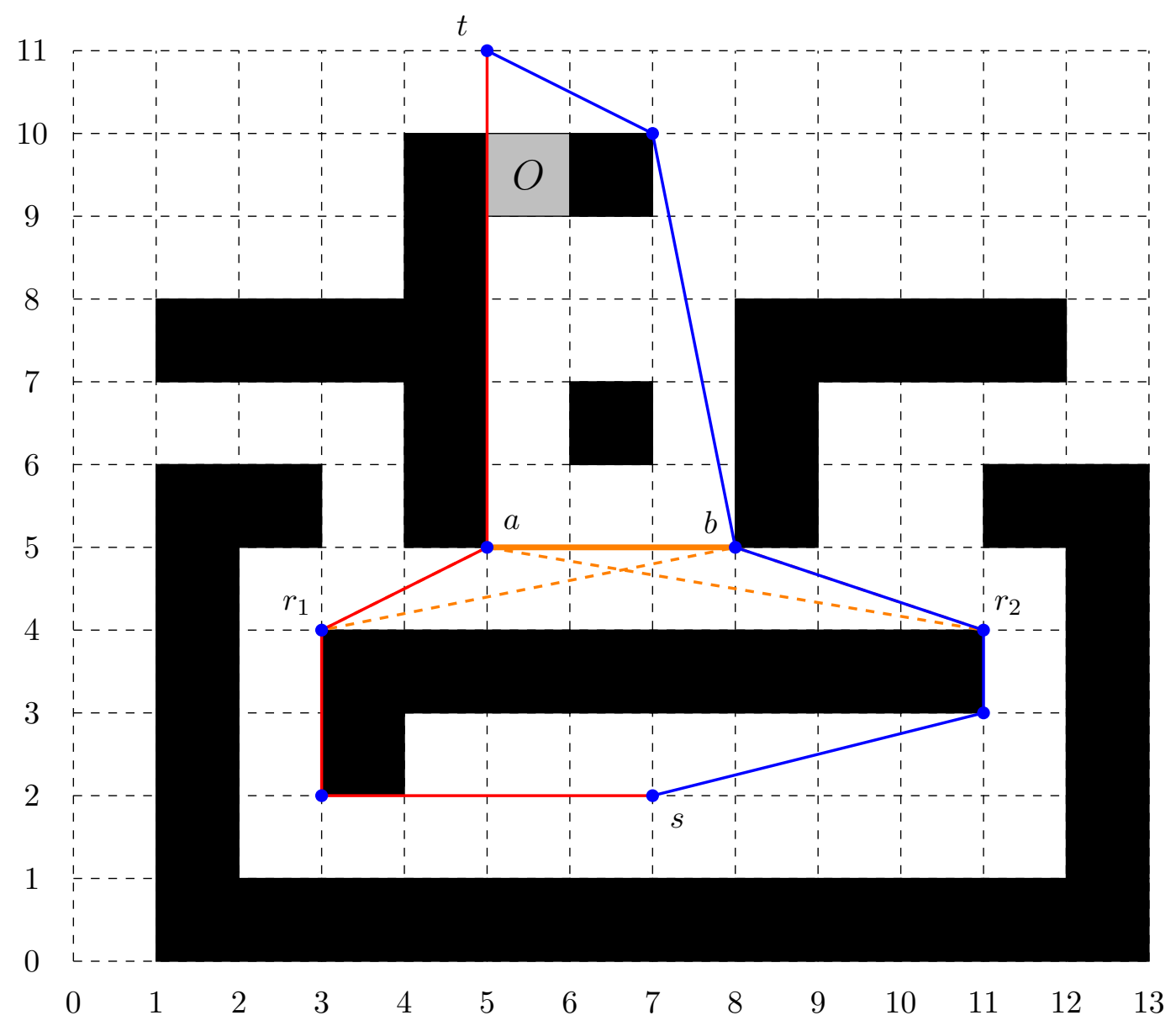

Figure 15: Illustrating that search nodes cannot be trivially pruned with search nodes $n_{1}=\left([a, b], r_{1}\right)$ and $n_{2}=\left([a, b], r_{2}\right)$ : if $O$ is not an obstacle the optimal path between $s$ and $t$ goes through $n_{1}$ (red); otherwise it goes through $n_{2}$ (blue).

\section{Experimental Setup}

We conduct experiments on seven benchmark problem sets taken from Nathan Sturtevant's well known repository (Sturtevant, 2012). Three of the benchmarks originate from popular computer games and often appear in the literature. They are: Baldur's Gate II , Dragon Age Origins and StarCraft. The maps in these benchmarks vary in size; from several thousand nodes up to several million. The remaining four benchmarks comprise grids of size $512 \times 512$ with randomly placed obstacles of varying densities, from $10 \%$ to $40 \%$. Table 1 gives an overview of the benchmark problems. We give the number of maps and instances per problem set and a distribution for the number of node expansions required by a reference algorithm, $\mathrm{A}^{*}$ using an octile distance heuristic ${ }^{7}$, to solve all problems in each benchmark set. The latter metric gives us a baseline for comparing the "difficulty" of problems appearing in each benchmark set.

7. Octile distance is analogous to Manhattan distance but generalised to 8-connected grids. 


\begin{tabular}{|r|r|r|r|r|r|r|r|r|r|}
\hline Benchmark & \#Maps & \#Instances & \multicolumn{7}{|c|}{ Nodes Expanded by A* } \\
\cline { 4 - 9 } & & & Min & Q1 & Median & Mean & Q3 & Max & StDev \\
\hline Baldur's Gate II & 75 & 93160 & 2 & 166 & 2019 & 6302 & 9170 & 86720 & 9136 \\
Dragon Age & 156 & 159465 & 1 & 622 & 5880 & 14080 & 19150 & 126800 & 19744 \\
StarCraft & 75 & 198230 & 3 & 4808 & 26840 & 50000 & 70110 & 578900 & 63507 \\
Random 10\% & 10 & 16770 & 2 & 239 & 548 & 1886 & 1485 & 59280 & 3921 \\
Random 20\% & 10 & 17740 & 3 & 749 & 3869 & 8606 & 14680 & 53760 & 9905 \\
Random 30\% & 10 & 19200 & 4 & 3520 & 14190 & 20290 & 33710 & 96090 & 19162 \\
Random 40\% & 10 & 35360 & 3 & 12520 & 42850 & 51920 & 83770 & 169900 & 43558 \\
\hline
\end{tabular}

Table 1: An overview of the seven benchmark problems used in our experiments. We give the number of maps and problem instances in each benchmark and the distribution of nodes expanded by a reference algorithm $\left(\mathrm{A}^{*}\right)$ when solving all problems in each benchmark set.

We compare our purely online and optimal Anya algorithm with a number of state-ofthe-art any-angle techniques. These are: Theta* (Nash et al., 2007), Lazy Theta* (Nash, Koenig, \& Tovey, 2010), Field A* (Uras \& Koenig, 2015a) and an any-angle variant of two-level Subgoal Graphs (SUB-TL) (Uras \& Koenig, 2015b). All of these approaches are near-optimal and are not guaranteed to return the shortest path. The methods Theta*, Lazy Theta* and Field A* are all purely online. Only SUB-TL relies on an offline pre-processing step to further improve the performance of search. We use $\mathrm{C}++$ implementations for each of these algorithms; the source codes are made publicly available by Uras and Koenig (2015a).

Anya is implemented in Java and executed on JVM 1.8. To allow for comparisons across different implementation languages we use the A* algorithm (Hart, Nilsson, \& Raphael, 1968), implemented in both $\mathrm{C}++$ and Java, as a reference point ${ }^{8}$. We compare the performance of Anya against the Java implementation of $\mathrm{A}^{*}$ and all other algorithms with the $\mathrm{C}++$ implementation of $\mathrm{A}^{*}$. All experiments are performed on a $3 \mathrm{GHz}$ Intel Core i7 machine with $8 \mathrm{~GB}$ of RAM and running OSX 10.8.4. Source code for our implementation of Anya is available from https://bitbucket.org/dharabor/pathfinding.

\section{Results}

We evaluate performance using three different metrics: search time, nodes expanded and path length. All results are presented relative to a benchmark algorithm, A*, which we combine with a standard octile distance heuristic. For example, when comparing search time or nodes expanded, we will give figures for the relative speedup of each algorithm vs A* $^{*}$ Under this paradigm a search time speedup of 2 means twice as fast while a node expansion speedup of 2 means half as many nodes were expanded. When comparing path length we give the percent improvement in path length vs $A^{*}$. In all cases higher is better.

8. The $\mathrm{C}++$ implementation is due to Uras and Koenig (2015a); the Java implementation is our own. 


\begin{tabular}{|c|c|c|c|c|c|c|c|c|c|c|}
\hline \multirow[t]{2}{*}{ Benchmark } & \multicolumn{5}{|c|}{ Avg. Node Expansion Speedup } & \multicolumn{5}{|c|}{ Avg. Path Length Improvement (\%) } \\
\hline & Anya & Theta* $^{*}$ & L.Theta* & F.A* & SUB-TL & Anya & Theta* $^{*}$ & L.Theta* & F.A* & SUB-TL \\
\hline Baldur's Gate II & 91.13 & 1.95 & 1.96 & 1.01 & 907.10 & $4.65 \%$ & $4.62 \%$ & $4.61 \%$ & $4.38 \%$ & $4.58 \%$ \\
\hline Dragon Age & 19.60 & 1.05 & 1.05 & 0.90 & 57.45 & $4.34 \%$ & $4.27 \%$ & $4.22 \%$ & $4.05 \%$ & $4.28 \%$ \\
\hline StarCraft $40 \%$ & 40.73 & 1.27 & 1.27 & 0.95 & 166.00 & $5.02 \%$ & $4.95 \%$ & $4.92 \%$ & $4.70 \%$ & $4.88 \%$ \\
\hline Random $10 \%$ & 0.80 & 2.34 & 2.38 & 1.14 & 6.60 & $4.77 \%$ & $4.63 \%$ & $4.58 \%$ & $3.83 \%$ & $4.59 \%$ \\
\hline Random $20 \%$ & 0.77 & 1.23 & 1.17 & 0.80 & 2.56 & $4.57 \%$ & $4.34 \%$ & $4.15 \%$ & $3.26 \%$ & $4.30 \%$ \\
\hline Random $30 \%$ & 1.06 & 0.82 & 0.75 & 0.64 & 1.68 & $4.44 \%$ & $4.12 \%$ & $3.77 \%$ & $3.12 \%$ & $4.03 \%$ \\
\hline Random $40 \%$ & 2.20 & 0.90 & 0.86 & 0.82 & 2.40 & $4.14 \%$ & $3.95 \%$ & $3.48 \%$ & $3.22 \%$ & $3.74 \%$ \\
\hline
\end{tabular}

Table 2: We compare the performance of each algorithm in terms of average node expansion speedup and average path length improvement. Both metrics are taken with respect to a reference algorithm $\left(\mathrm{A}^{*}\right)$. In both cases higher is better.

We begin with Table 2 which shows average performance figures for nodes expanded and path length on each of our seven benchmark problem sets. We make the following observations:

- Anya is the best of the four purely-online algorithms, expanding fewer nodes in five of the seven benchmarks. On the three benchmarks drawn from real computer games Anya expands one order fewer nodes, on average, than its nearest purelyonline contemporary. Only the pre-processing-based SUB-TL algorithm expands fewer nodes, on average.

- Anya, as with all methods in our comparison, struggles to achieve a speedup on the four random benchmarks. In two of the four cases its performance is below that of the reference A* algorithm. Again, only the pre-processing-based SUB-TL algorithm is able to achieve a consistent, though much reduced, node expansion speedup.

- Anya, being optimal, shows the best improvement in path length; however all algorithms in our comparison are very close to optimal, on average.

Next, we evaluate performance in terms of search time. Rather than taking a simple average on a per benchmark basis (or across all benchmarks) we instead sort instances according to difficulty, as measured by the number of node expansions required for the reference $A^{*}$ algorithm to solve each problem. This approach gives a more holistic overview of performance and reduces the effect of any bias associated with the selection of instances that comprise each benchmark set ${ }^{9}$. Results from this analysis are given in Figure 16 . We make the following observations:

- Anya is often more than one order of magnitude faster than the reference $\mathrm{A}^{*}$ algorithm on the benchmarks drawn from real computer games. Performance is mixed on the four random benchmarks, with all evaluated methods struggling to achieve a speedup.

9. As per Table 1, problem instances that can be regarded as "easy" often outnumber instances that can be regarded as "hard". These difference have the effect of skewing performance indicators that are computed as simple averages over all instances in each benchmark set. 



Random; $512 \times 51230 \%$ obstacles
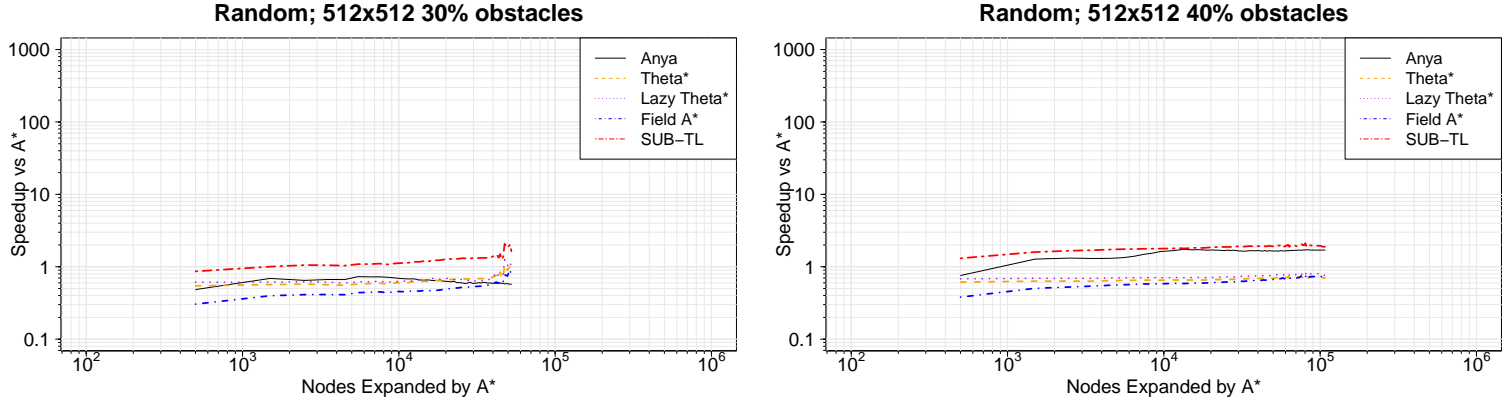

Figure 16: Search time speedup. We compare performance on each of our seven benchmarks in terms of search time. Figures are given as relative speedup vs. a reference $A^{*}$ algorithm. Problem instances are sorted by difficulty using $A^{*}$ node expansion rank. Note that each plot is log-log. 
- Anya is the fastest of the four purely online methods under evaluation. Its performance is often comparable with the pre-processing based SUB-TL technique and, on particularly challenging instances from the StarCraft domain, Anya is non-dominated ${ }^{10}$.

- Anya's performance in terms of search time is less than the value suggested by the (previously evaluated) node expansion metric. This reflects the fact that each node expansion made by Anya involves analysing the grid; looking for roots and searching for intervals.

\subsection{Discussion}

We have seen that Anya compares well with current state-of-the-art any-angle pathfinding algorithms. In an (almost) apples-to-apples comparison with three contemporary and purely online search technique (Theta*, Lazy Theta* and Field A*) we have seen that Anya usually expands fewer nodes per search and terminates up to one order of magnitude faster. These results are further underscored by the fact that Anya is the only online algorithm that is guaranteed to return a Euclidean-optimal path. We may surmise that, in many cases and applications, Anya appears preferable to each of these alternative algorithms.

Next, we make an apples-to-oranges comparison between the purely online Anya algorithm and the near-optimal and offline enhanced SUB-TL algorithm. We have seen that while Anya is usually not as fast as SUB-TL its performance is sometimes comparable. Moreover, Anya retains an advantage when solving especially challenging instances drawn from real computer games. SUB-TL appears to be preferable to Anya in cases where additional space and time is available to create and store its associated subgoal graph or in cases where such overheads can be amortised over many online instances. When extra space and time is not available, or in cases where the map is subject to change (e.g. new obstacles are added or existing obstacles are removed), Anya appears to be preferable to SUB-TL.

The main strength of Anya is that it searches over sets of nodes from the grid rather than considering individual locations one at a time. Expansion can thus be considered as a macro operator, meaning that Anya bears some similarity to speedup techniques using hierarchical abstraction; e.g. HPA* (Botea et al., 2004). An important difference is that Anya constructs its abstract graph on-the-fly rather than as part of a pre-processing step.

One current drawback associated with Anya is that nodes can contain overlapping intervals. This occurs when an interval is reachable from two different root points, neither of which can be pruned (e.g. when both root locations are reached for the first time; as illustrated in Figure 15). Such nodes are, either in part or in whole, redundant and provided their $f$-value is smaller than the optimal distance to the goal — will themselves beget yet more redundant successors. We can see this behaviour especially in the results for benchmarks Random $10 \%$ and Random $20 \%$ where SUB-TL achieves a speedup of several factors while Anya struggles to maintain parity with the reference A* algorithm. It seems reasonable to improve the current algorithm by attempting to identify such overlaps in order to prune them from consideration. An efficient and effective algorithm for achieving this goal is the subject of further work.

10. In the Pareto sense; i.e. there are problem instances where Anya is better than SUB-TL according to some metric of interest such as node expansions or search time 


\section{Related Work}

Among the simplest and most popular approaches for solving the any-angle pathfinding problem is string-pulling. The main idea is to find a path on the input grid map, often using some variant of $\mathrm{A}^{*}$ (Hart et al., 1968), and then post-process that path in order to remove unnecessary turning points. Several such methods have appeared in the literature of Game Development; e.g. see the work of Pinter (2001) and Botea et al. (2004).

A number of algorithms improve on string-pulling by interleaving node expansion and path post-processing during online search. Particular examples include Field D* (Ferguson \& Stentz, 2005) and Field A* (Uras \& Koenig, 2015a), both of which use linear interpolation to smooth grid paths one cell at a time, and Theta* (Nash et al., 2007), which introduces a shortcut each time a successful line-of-sight check is made; from the parent of the current node to any of its successors. Though still sub-optimal in many cases such approaches are nevertheless attractive for being able to search purely online and for being efficient in practice. In addition to the two examples given there are numerous other works, often appearing in the literature of Artificial Intelligence, that apply and improve on the basic interleaving idea. We refer the interested reader to Nash \& Koenig, 2013 for a recent survey and overview.

Accelerated A* (Šišlák, Volf, \& Pěchouček, 2009) is an online any-angle algorithm that is conjectured to be optimal but for which no strong theoretical argument is made. Similar to Theta*, it differs primarily in that line-of-sight checks are performed from a set of expanded nodes rather than a single ancestor. The size of the set is only loosely bounded and, for challenging problems, can include a large proportion of nodes on the closed list.

One recent and successful line of research involves the combination of string-pulling with an offline pre-processing step. Such works are compelling because they can significantly improve on the performance of purely online search; not just in terms of solution quality but also running time. Block A* (Yap, Burch, Holte, \& Schaeffer, 2011) is one such example. This sub-optimal algorithm pre-computes a database of Euclidean-optimal distances in all possible tile configurations of a certain size (e.g. all possible $3 \times 3$ blocks). The database obviates the need for explicit visibility checks or indeed any type of online string-pulling. The pre-processing step needs to be performed exactly once; the database remains valid if the tiles on the map change or indeed if the map itself changes entirely. Another recent work improves on Theta* by combining that algorithm with a pre-processing based graph abstraction technique (Uras \& Koenig, 2015b). This approach, referred to in Section 9 as SUB-TL, is shown to improve on both the running time and solution quality of Block $\mathrm{A}^{*}$. The main disadvantage (vs. Block $\mathrm{A}^{*}$ ) is that the abstract graph needs to be re-computed or repaired each time the map changes.

The Euclidean Shortest Path Problem is a well known and well researched topic in the areas of Computational Geometry and Computer Graphics. It can be seen as a generalisation of the Any-angle Pathfinding Problem. It asks for a shortest path in a plane but does not impose any restrictions on obstacle shape or obstacle placement (cf. grid aligned polygons made up of unit squares).

Visibility graphs (Lozano-Pérez \& Wesley, 1979) are a family of well-known and popular techniques for optimally solving the Euclidean Shortest Path Problem. Searching in such graphs requires $O\left(n^{2} \log _{2} n\right)$ time but the approach can be much faster in practice. There 
are two main disadvantages: (i) computing the graph requires an offline pre-processing step and $O\left(n^{2}\right)$ space to store; (ii) the graph is static and must be recomputed or repaired if the environment changes. More sophisticated variants such as Tangent Graphs (Liu \& Arimoto, 1992) and Silhouette Points (Young, 2001) are particularly efficient variants of visibility graphs but the same disadvantages apply.

Another family of exact approaches for solving the Euclidean Shortest Path Problem is based on the Continuous Dijkstra paradigm (Mitchell et al., 1987). The most efficient of these algorithms (Hershberger \& Suri, 1999) involves a pre-computation requiring $O\left(n \log _{2} n\right)$ space and $O\left(n \log _{2} n\right)$ time. The result is a Shortest Path Map; a planar subdivision of the environment that can be used to find a Euclidean shortest path in just $O\left(\log _{2} n\right)$ time; but only for queries originating at a fixed source. Like visibility graphs, this approach also introduces additional memory overheads (storing the subdivision) and the pre-processing step must be re-executed each time the environment or the start location changes.

\section{Conclusion}

We study any-angle pathfinding: a problem commonly found in the areas of robotics and computer games. The problem involves finding a shortest path between two points in a grid but asks that the path is not artificially constrained to the fixed points of the grid. The best known online algorithms for the any-angle problem, to date, all compute approximate solutions rather than optimal shortest paths. Additionally no online methods have been able to achieve a consistent speedup vs. the $\mathrm{A}^{*}$ algorithm - a common reference point for measuring performance in the literature. In this work we present a new online, optimal and practically efficient any-angle technique: Anya. Where other works obtain good performance by reasoning at the grid level our method considers sets of points from the grid which are taken together as contiguous intervals. This approach requires revisiting the classical definition of search nodes and successors and requires the introduction of a new technique for computing the $f$-value of each node. We give a thorough algorithmic description of this new search paradigm and we give theoretical arguments for its completeness and optimality preserving characteristics.

In an (almost) apples-to-apples comparison we evaluate Anya against three contemporary near-optimal and online techniques: Theta*, Lazy Theta* and Field A*. We show that, on a range of popular benchmarks, Anya is faster than each of these alternatives, all while guaranteeing to find an optimal shortest path. In an apples-to-oranges comparison we evaluate Anya against SUB-TL: a very fast pre-processing-based near-optimal any-angle technique. We show that Anya is non-dominated when compared to SUB-TL and even maintains an advantage on some particularly challenging instances drawn from real computer games. Another advantage is that, unlike SUB-TL, Anya does not assume the map is static; i.e. it can be readily applied to pathfinding problems involving dynamically changing terrain.

Any-angle pathfinding has received significant attention from the AI and Game Development communities but until now it has been an open question whether any optimal and online algorithm exists. Anya answers this question in the affirmative. 


\subsection{Future Work}

There are several possible directions for future work. Perhaps most obvious is the development of improvements and extensions to the current Anya algorithm. For example, we believe the empirical performance of Anya could be enhanced by generating successors nodes that do not contain any redundant (or partially redundant) intervals. One possibility is to keep a closed list of previously encountered intervals. A stronger variant of this idea involves bounding the $g$-value of grid intervals and only generating successor nodes when at least one point inside a candidate interval can be relaxed. A related and orthogonal improvement involves pre-processing the grid and identifying intervals apriori. This enhancement can speed up search by avoiding entirely all grid scanning and interval projection operations that are currently necessary in order to generate each node.

We have seen that reasoning over sets of points from the grid, rather than individual locations, is computationally beneficial. We believe the same type of search paradigm employed by Anya can be generalised to improve the performance of grid-optimal search in addition to any-angle pathfinding.

As a final suggestion for further work, we believe Anya might also be generalised to two-dimensional maps with arbitrarily shaped polygonal obstacles, rather than just grids. A benefit of this generalisation would be to avoid the discretisation of the world in which a path is searched for. This would even improve the quality of the path returned as the optimal any-angle path is often non optimal in the non-discretised version of the map.

\section{Acknowledgements}

We thank Tansel Uras for assistance with the source codes used in the experimental section of this paper. We also thank Adi Botea and Patrik Haslum for helpful suggestions during the early development of this work.

The work of Daniel Harabor and Alban Grastien is supported by NICTA. NICTA is funded by the Australian Government as represented by the Department of Broadband, Communications and the Digital Economy and the Australian Research Council through the ICT Centre of Excellence program.

The work of Dindar Öz and Vural Aksakalli is supported by The Scientific and Technological Research Council of Turkey (TUBITAK), Grant No. 113M489. 


\section{Appendix A.}

We provide additional details for the implementation of Anya's successor set generation algorithm. Our method depends on basic operations which are technically simple: grid scanning, traversability tests and linear projection operations. We do not attempt to reproduce the mechanical details of such operations. Instead we focus our presentation toward intuitive understanding of the overall process.

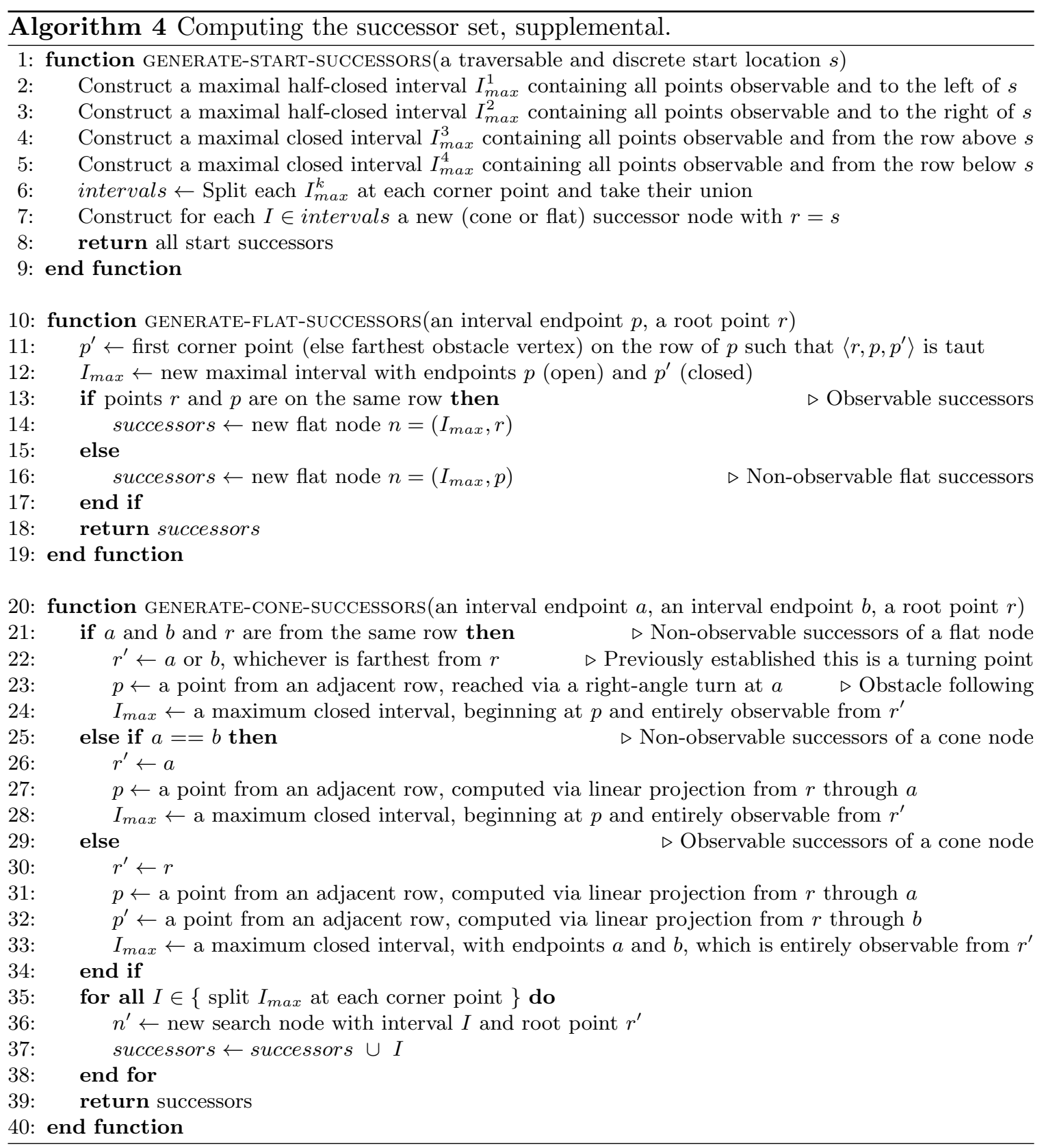




\section{References}

Björnsson, Y., \& Halldórsson, K. (2006). Improved Heuristics for Optimal Path-finding on Game Maps. In Proceedings of the Second Artificial Intelligence and Interactive Digital Entertainment Conference, June 20-23, 2006, Marina del Rey, California, pp. 9-14.

Botea, A., Müller, M., \& Schaeffer, J. (2004). Near Optimal Hierarchical Path-Finding. Journal of Game Development, 1(1), 7-28.

Ferguson, D., \& Stentz, A. (2005). Field D*: An Interpolation-based Path Planner and Replanner. In Robotics Research: Results of the 12th International Symposium, ISRR 2005, October 12-15, 2005, San Francisco, CA, USA, pp. 239-253.

Graham, R. L., Knuth, D. E., \& Patashnik, O. (1989). Concrete Mathematics - A Foundation for Computer Science. Addison-Wesley.

Harabor, D. D., \& Grastien, A. (2013). An Optimal Any-Angle Pathfinding Algorithm. In Proceedings of the Twenty-Third International Conference on Automated Planning and Scheduling, ICAPS 2013, Rome, Italy, June 10-14, 2013.

Harabor, D. D., \& Grastien, A. (2014). Improving Jump Point Search. In Proceedings of the Twenty-Fourth International Conference on Automated Planning and Scheduling, ICAPS 2014, Portsmouth, New Hampshire, USA, June 21-26, 2014.

Hart, P. E., Nilsson, N. J., \& Raphael, B. (1968). A Formal Basis for the Heuristic Determination of Minimum Cost Paths. IEEE Transactions on Systems Science and Cybernetics, 4(2), 100-107.

Hershberger, J., \& Suri, S. (1999). An Optimal Algorithm for Euclidean Shortest Paths in the Plane. SIAM Journal on Computing, 28(6), 2215-2256.

Liu, Y.-H., \& Arimoto, S. (1992). Path Planning Using A Tangent Graph For Mobile Robots Among Polygonal And Curved Obstacles. International Journal of Robotics Research, 11, 376-382.

Lozano-Pérez, T., \& Wesley, M. A. (1979). An Algorithm for Planning Collision-Free Paths Among Polyhedral Obstacles. Communications of the ACM, 22(10), 560-570.

Mitchell, J. S. B., Mount, D. M., \& Papadimitriou, C. H. (1987). The Discrete Geodesic Problem. SIAM Journal on Computing, 16(4), 647-668.

Nash, A., Daniel, K., Koenig, S., \& Felner, A. (2007). Theta*: Any-Angle Path Planning on Grids. In Proceedings of the Twenty-Second AAAI Conference on Artificial Intelligence, July 22-26, 2007, Vancouver, British Columbia, Canada, pp. 1177-1183.

Nash, A., \& Koenig, S. (2013). Any-Angle Path Planning. AI Magazine, 34(4), 9.

Nash, A., Koenig, S., \& Tovey, C. A. (2010). Lazy Theta*: Any-angle Path Planning and Path Length Analysis in 3D. In Proceedings of the Twenty-Fourth AAAI Conference on Artificial Intelligence, AAAI 2010, Atlanta, Georgia, USA, July 11-15, 2010.

Pinter, M. (2001). Toward More Realistic Pathfinding. Game Developer Magazine, 8(4). 
Šišlák, D., Volf, P., \& Pěchouček, M. (2009). Accelerated A* Trajectory Planning: Gridbased Path Planning Comparison. In 4th ICAPS Workshop on Planning and Plan Execution for Real-World Systems.

Sturtevant, N. (2012). Benchmarks for Grid-Based Pathfinding. Transactions on Computational Intelligence and AI in Games, 4(2), 144 - 148.

Sturtevant, N. R., \& Bulitko, V. (2011). Learning Where You Are Going and from Whence You Came: h- and g-Cost Learning in Real-Time Heuristic Search. In 22nd International Joint Conference on Artificial Intelligence, IJCAI 2011, pp. 365-370.

Sun, X., Yeoh, W., Chen, P.-A., \& Koenig, S. (2009). Simple Optimization Techniques For A*-based Search. In 8th International Joint Conference on Autonomous Agents and Multiagent Systems, AAMAS 2009, Budapest, Hungary, May 10-15, 2009, Volume 2, pp. 931-936.

Uras, T., \& Koenig, S. (2015a). An Empirical Comparison of Any-Angle Path-Planning Algorithms. In Proceedings of the Eighth Annual Symposium on Combinatorial Search, SOCS 2015, 11-13 June 2015, Ein Gedi, the Dead Sea, Israel, pp. 206-211.

Uras, T., \& Koenig, S. (2015b). Speeding-Up Any-Angle Path-Planning on Grids. In Proceedings of the Twenty-Fifth International Conference on Automated Planning and Scheduling, ICAPS 2015, Jerusalem, Israel, June 7-11, 2015, pp. 234-238.

Yap, P., Burch, N., Holte, R. C., \& Schaeffer, J. (2011). Block A*: Database-Driven Search with Applications in Any-Angle Path-Planning. In Proceedings of the Twenty-Fifth AAAI Conference on Artificial Intelligence, AAAI 2011, San Francisco, California, USA, August 7-11, 2011.

Young, T. (2001). Optimizing Points-of-Visibility Pathfinding. In Game Programming Gems 2, pp. 324-329. Charles River Media. 